\title{
LOOP-VOLTage TOMOgRaPhy IN TOKaMaks USing Transient Synchrotron Radiation
}

\author{
\. J. Fisch ${ }^{\dagger}$ and A. H. Fritz \\ ${ }^{\dagger}$ Princeton Plasma Physıcs Laboratory, Princeton University, Princeton. $\mathrm{YJ} 08543$ \\ $\ddagger$ Department of Physics, Hunter College, sew York, NY 10021
}

\begin{abstract}
The loop voltage in tokamaks is particularly difficult to measure ar: where but at the plasma periphery. A brief, deliberate, perturbation of hot plasma electrons, however. produces a transient radiation response that is sensitive to this voltage. $I$ - investigate how such a radiation response can be used to diagnose the loop voltage.
\end{abstract}

\section{DLSCLAIMER}

\begin{abstract}
This report was prepared as ap acoount of work sponsored by an ajency of the United Sutes Government. Neither the United States Government nor any agency thereof, nor any' of their employes, makes any warranty, express or implied, or assumes any legal liability br responsibility for the accuracy, compleieness, of usefulness of any informalion, apparatus, product, or process disclosed, or represents that its use would nor in [ringe privately awned ights. Reference herein to any specifie commercial product, process, or service by trade name. 1radematk, manulacturer, or etherwise does not necessarily constitute or imply its endorsement, recommendation, of lavoring by the United Stales Government or any agency thereof. The wiews and opinions of authors expressed herein do not necessarily state or reflect those of the United States Governmeml or any agency thereof
\end{abstract}




\section{Introduction}

An examination of the transient. synchrotron radiation signal which urises from a deliberate. perturbation of hot tokamak electrons, can be quite informative. The perturbation might be produced, for example, through brief heating of superthermal electrons by lower-hybrid waves. The plasma radiation response to this perturbation. in frequency-time space, forms a two-dimensional pattern that looks different under different plasma conditions. An example of this radiation pattern, $R(\omega, t)$, is given in Fig. 1. The parameters to which this radiation is sensitive include the dc electric field $E$, the ion charge state $Z_{\text {eff }}$. the angle of riewing with respect to the magnetic field $\theta$, the density $n$. and the precise velocity of the perturbed electrons. These parameters comprise a set of conditions under which the radiation response is observed. Through a comparison of the radiation patterns that would be produced with any parameter sets that might possibly explain the transient signal, the relative probabilities of the competing parameter sets can be evaluated.

The deliberate heating or probing of the plasma to produce synchrotron radiation directly attributable to this probe has been the subject of previous work. A mathematical inversion of the two-dimensional transient synchrotron data to obtain a two-dimensional electron momentum distribution function was described by FISCH (1988), assuming as given, however, parameters describing the plasma. Of greater diagnostic interest is that the deliberately produced transient emissions could be employed to deduce various plasma parameters, as shown by Fisch AND KRITZ (1989a). In these previous works, the de electric field was assumed absent.

A generalization of this work to include a dc parallel electric field (FISCH AND KRITZ, 1989 b) both expands the range of experiments for which the radiation can be used to deduce other parameters and allows us to deduce the electric field itself, something entirely unavailable otherwise. Typically less than a volt/meter in a tokamak, this field is far too small to be inferred through atomic phenomena, and cannot be measured directly by probes because the plasma is too hot. Its effect is manifest, however, in the dynamics of superthermal electrons, exactly those that syachrotron radiate most profusely. Of course, in a truly steady state, one could measure the loop voltage at the plasma edge to deduce the central loop voltage, but in many instances the plasma is far from steady state. An example for which the edge voltage may not be informative is during nonohmic currentdrive on axis. The plasma response at the periphery reflects the current-drive only after a magnetic relaxation time. Early experiments on current-drive by lower-hybrid waves, in fact, were ambiguous precisely because the experiments were coo short.

A goal of this paper is to simulate how from a transient radiation signal, one might detect a loop voltage on axis at the same time that the loop voltage vanishes at the plasma periphery. Additionally, in this paper. we derive in detail the Green's function for the radiation response for relativistic electrons in the presence of the dc parallel electric field. We extend here two themes of our previous work: first. that the transient response indeed informs on plasma parameters. and. moreover. that the parameters of interest can be deduced almost orthogonally, i.e., ignorance or even misinformation concerning some parameters does not impair significantly the inference of other parameters: and second. that fast algorithms are available for processing the data quickly, which is an important requirement when searching a large dimensional space. 
The technique exploits a fortuitous separation of time scales $1 / \omega \ll \tau_{\text {del }} \ll T_{c} \ll$ par. From the first inequality we have that the radiation frequency $\sim(\sim 100 \mathrm{GH} z)$ is sufficiently characterized on the instrumental detection time scale of $\tau_{\text {det }}$, which can be $50 \mu \mathrm{s}$. The time history of the radiation response is well-characterized since there are many detector observations in a superthermal electron slowing down time, $\tau_{c}$. yet. the parameters themselves change on the longer time scale $r_{\text {par }}$, so that their values may be treated as constant during the transient analysis. Of course, there is the spportunity. by repeating the probe, to average the results of several transient analyses.

By producing a transient signal, we endow the time measurements with informative potential otherwise absent. For example, the steady background synchrotron radiation is entirely insensitive to the ion charge state $Z_{\text {eff }}$; no matter how frequently measurements are made, this parameter is not deducible from the background radiation. However. the transient response is quite sensitive to this parameter, since this parameter governs the collisional pitch-angle scattering of fast electrons in velocity space. Synchrotron radiation is quite sensitive to the velocity-space pitch-angle of radiating electrons.

Throughout this work, we distinguish the total radiation ernitted from the plasma into angle $\theta, R_{\text {tot }}(\omega, t ; \theta)$, from the incremental or transient signal. $R(\omega, t ; \theta)$, that is directly proportional to the invasive, brief heating that we refer to as the probe heating. The total radiation emitted from an optically thin plasma is

$$
R_{\mathrm{tot}}(\omega, t ; \theta)=\int d^{3} p f(\mathrm{p}, t) I(\omega, \mathrm{p} ; \theta)
$$

where $f$ is the electron momentum distribution function and $I$ is the radiation power at frequency $\omega$, due to a single elect:on at momentum $p$, which is radiated into angle $\theta$ with respect to the magnetic feld. The radiation can be considered separately at either the ordinary or extraordinary wave polarization.

The distribution function $f$ can be separated into $f=f_{M}\left(1+\phi_{B}+\phi\right)$, where $f_{M} i$ a Maswellian distribution, $\phi_{\mathrm{B}}$ describes the relatively constant deviation from Max ali, an of the background distribution, and $\phi$ describes the time-dependent distribution specilically associated with the probe heating. In terms of contributing to the collision integral, both $\phi_{\mathrm{B}}$ and $\phi$ may be treated as srnall, so that $f$ obeys the linearized Fokker-Planck equation. The evolution of $\phi$ may then be written as

$$
f_{M} \partial \phi / \partial t+q \mathrm{E} \cdot \nabla_{\mathrm{p}} f_{\mathrm{M}} \phi-C(\phi)=0
$$

where $C$ is a collision term. The initial condition on $\phi$, which is the result of the probe heating, is taken to be $f_{M} \phi=-Q(p / m c) /(m c)^{3}$, where $m$ is the electron mass. where the speed of light, $c_{i}$ is introduced for later normalization. and where $Q$ is the normalized initial deviation from background due to the probe heating. For example, were the probe to consist of an impulse of a narrow spectrum of high-phase-velocity lower-hybrid waves. then $Q(\mathbf{p} / m c)$ would be finite in a narrow range of superthermal $\mathbf{p}$. Using normalized momentum, $u=p / m c$. and normalized time, $\tau=\nu_{c} t$, with collision frequency $\nu_{c}=n q^{4} \log \Lambda / 4 \pi m^{2} \epsilon_{0}^{2} c^{3}$, we write the incremental radiation associated with the initial condition on $f_{M} \odot$ as

$$
R(\omega, \tau ; \theta)=\int d^{3} p f_{M} \phi(p, \tau) I(\mu, p ; \theta)=\int d^{3} u(\cdot(\alpha, \mathbf{u}, \tau ; \theta) Q(\mathbf{v})
$$


where the second equality above recognizes that a large savings in effort is possible by defining a Green's function $v$ for the radiation response.

The comparison of many possible radiation responses to data is facilitated by fast algorithms. The Green's function makes efficient the simultaneous consideration of many initial perturbations $Q(\mathbf{p})$. Choosing to perturb electrons on the tail of the distribution function. superthermal but not runaways, makes possible enormous analytic progress in solving for $\psi$, since for these electrons, energy diffusion by collisions is ignorable compared to energy loss. Moreover. Eqs. (1.2) and (1.3) admit several scale-invariant transformations of the radiation response $R(\omega . t)$. Having solved for $R(\omega, t ; \Theta)$, where $\Theta$ is a set of parametric dependencies which includes the magnetic field amplitude $B$, electric field $E$. the density $n_{1}$ and the perturbation amplitude $A$, we also have for any constants $\alpha_{1}, \alpha_{2}$. and $a_{3}$,

$$
R\left(\omega, t ; \alpha_{1} B, \alpha_{2} A, \alpha_{3} \eta, E\right)=\alpha_{1} \alpha_{2} R\left(\omega / \alpha_{1}, t / \alpha_{3} ; B, A, n, E / \alpha_{3}\right) .
$$

Further simplification of $\mathrm{Eq} .(4)$ is made possible by choosing to heat those electrons for which it is permissible to linearize $R=R_{\theta}+E R_{1}$. These would be tail electrons, but not nearly so fast as to be runaway electrons that are strongly affected by the dc field.

The use of the background emission is, of course, an established diagnostic for the electron temperature, and recently there have been attempts to uncover further details of the electron momentum distribution function. Useful constraints on the electron distribution function have been derived, e.g., by CELATA AND BOYD (197T), TAMOR (1979), Boriatici et al. (1983), Celata (1985), Huchinson and Kato (1986), Kato ajd HUCHINSON (1986), and LUCE et al. (1988). In a relativistic electron ring geometry. a one-dimensional $f$ was deduced elegantly by MAHAJAN et al. (1974). In these studies, the deduction of details of the electron distribution function was based on the synchrotion emission from the entire distribution of electrons. The utility of information provided by transient radiation during collisional relaxation has been recognized before: for exampie. ALIKAEV et al. (1976) observe radiation decay subsequent to intense cyclotron heating in the TM-3 tokamak, and GIRuzzI et al. (1986) observe numerically the transient radiation pattern associated with cyclotron heating in the presence of a dc electric field. A more refined treatment, but with no dc field, was recently carried out by GIRU2zI (1988).

In our work there is a great emphasis on fast computation in extracting important information from transient signals. In Sec. 2, we derive the Green's function $\psi$ for the transient radiation response in closed form and in terms of integrals over elementary functions. This efficient form enormousiy simplifies the work of comparing numerically radiation responses arising under different plasma conditions. Certain details of the derivation are left to Appendices A and B. In Sec. 3, we examine the utility of the method in the nontrivial parameter space of six unknowns. Here, radiation patterns arising from over a million parameter sets are compared to numerically simulated noisy data. The toise model is specified. so precise statements can be derived concerning the probability distribution of parameter sets in view of the simulated data. In Sec. 4, we apply information-theoretic techniques to assess both the informative content of the transient synchrotron data and the relative orthogonality of parameters of interest. In Sec. 5, we summarize our findings and we offer a number of caveats regarding the applicability of the technique. 


\section{Green's Function for the Radiation Response}

The Greer.'s function for the radiation response, w. solves the relativistic FokkerPlanck adjoint equation (see e.g., FISCH, 1987), which we write as

$$
f_{\mathrm{N}} \partial v / \partial t-q f_{\mathrm{M}} \mathrm{E} \cdot \nabla_{\mathrm{p}} \psi-C(\omega)=0 .
$$

We employ the normalized variables, $\mathbf{u}=\mathbf{p} / m c$. $\tau=\nu_{c} t$. and $\mathcal{E}=q E / m c \nu_{c}$. and we specialize to superthermal excitation in the high-velocity limit. in order to write the adjoint equation as

$$
\frac{\partial \psi}{\partial \tau}-\mathcal{E} \frac{\partial \psi}{\partial u_{\|}}+\frac{1}{u^{3}}\left(\gamma^{2} u \frac{\partial \psi}{\partial u}-\gamma \frac{1+Z_{\mathrm{eff}}}{2} \frac{\partial}{\partial \mu}\left(1-\mu^{2}\right) \frac{\partial}{\partial \mu} \psi\right)=0 .
$$

where $Z_{\text {eff }}$ is the ion charge state and we defined $\gamma^{2}\left(u j \equiv 1+u^{2}\right.$ and $\mu \equiv p_{\|} / p$. This equation is to be solved with the initial condition $\psi(\omega, \mathbf{u}: \theta, \tau=0)=I(\omega, \mathbf{u}: \theta)$. The solution for $\psi$ is then used in Eq. (1.3).

We are fortunate that Eq. (2.1) is tractable analytically in the limit of small electric fields, i.e., for small $\mathcal{E}$. Let us expand

$$
\psi=\psi^{(0)}+\mathcal{E} \dot{\psi}^{(1)}+\mathcal{E}^{2} \psi^{(2)}+\cdots
$$

then the equation for $\psi^{(0)}$ becomes

$$
\frac{\partial \psi^{(0)}}{\partial \tau}+\frac{1}{u^{3}}\left(\gamma^{2} u \frac{\partial \psi^{(0)}}{\partial u}-\gamma \frac{1+Z_{\mathrm{eff}}}{2} \frac{\partial}{\partial \mu}\left(1-\mu^{2}\right) \frac{\partial}{\partial \mu} \psi^{(0)}\right)=0,
$$

with initial condition $\psi(\omega, \mathbf{u} ; \theta, \tau=0)=I(\omega, \mathbf{u} ; \theta)$. The equations for higher arder $\psi^{(i)}$ are inhomogeneous but with homogeneous initial conditions. The equation for $\psi^{(1)}$ becomes

$$
\frac{\partial \psi^{(1)}}{\partial \tau}+\frac{1}{u^{3}}\left(\gamma^{2} u \frac{\partial \psi^{(1)}}{\partial u}-\gamma \frac{1+Z_{\text {eff }}}{2} \frac{\partial}{\partial \mu}\left(1-\mu^{2}\right) \frac{\partial}{\partial \mu} \psi^{(1)}\right)=\varepsilon \frac{\partial \psi^{(0)}}{\partial u_{\|}}
$$

to be solved with a homogeneous initial condition.

The method of characteristics may be used to integratie $\mathrm{E}_{\mathrm{q}} \cdot(2.2)$ for $\psi^{(0)}$, once we separate both $\psi^{(0)}$ and the initial condition into Legendre hanionics, $P_{k}(\mu)$. Let

$$
\begin{gathered}
w_{k}^{(0)}=\frac{2}{2 k+1} \int_{-1}^{1} P_{k}(\mu) \psi^{(0)} d \mu, \\
I_{k}=\frac{2}{2 k+1} \int_{-1}^{1} P_{k}(\mu) I(\mu \cdot \mathbf{u} ; \theta) d \mu,
\end{gathered}
$$

then the equation for $w_{k}^{(0)}$ can be written as

$$
\frac{\partial w_{k}^{(0)}}{\partial \tau}+\frac{\gamma^{2}}{u^{2}} \frac{\partial u_{k}^{(0)}}{\partial u}+\alpha_{k} \frac{\gamma}{u^{3}} u_{k}^{(0)}=0
$$


where $\alpha_{k} \equiv\left(1+Z_{\text {eff }}\right) k(k+1) / 2$. Let $u=u(s, x), T=T(s, x)$, where $s$ measures along the characteristics and $x$ specifies the characteristic. The initial conditions, specified on $s=0$. are $v_{k}^{(0)}[u(0, x), \tau(0, x)]=\psi_{k}^{(0)}(0, x)$, where we can parameterize the initial conditions by writing $\tau(0, x)=0, u(0, x)=x$, and $\psi_{k}^{(0)}(0, x)=I_{k}(x: \omega, \theta)$. Then the characteristic equations, and their solutions for the given initial conditions, are:

$$
\begin{gathered}
\frac{\partial \tau}{\partial s}=1 \Longrightarrow \tau=s . \\
\frac{\partial u}{\partial s}=\frac{\gamma^{2}}{u^{2}} \Longrightarrow \quad \frac{u^{\prime 2}}{1+u^{\prime 2}} d u^{\prime}=s . \\
\frac{\partial v_{k}^{(0)}}{\partial s}=-\frac{\gamma}{u^{3}} \alpha_{k} \psi_{k}^{(0)} \Longrightarrow \psi_{k}^{(0)}=I_{k}(x)\left[\frac{1+\gamma(u)}{u}\right]^{\alpha_{k}} /\left[\frac{1+\gamma(x)}{x}\right]^{\alpha_{k}} .
\end{gathered}
$$

It remains to give explicitly the function $x(\tau, u)$. We define a function

$$
g(u) \equiv \int_{0}^{u} \frac{u^{\prime 2}}{1+u^{\prime 2}} d u^{\prime}=u-\tan ^{-1} u,
$$

then. we have $g(u)-g(x)=s=\tau$, and, defining also an inverse function. $g^{-1}$, we have

$$
x=g^{-1}[g(u)-\tau]
$$

Thus, substituting for $x(u, \tau)$ in Eq. (2.5c), we have the zeroth order Green's function $\psi_{k}^{(0)}(u, T)$.

The solution to the first order adjoint equation can be approached similarly. We separate $\psi^{(1)}$ into Legendre harmonics to derive for the kth harmonic,

$$
\frac{\partial \psi_{k}^{(1)}}{\partial \tau}+\frac{\gamma^{2}}{u^{2}} \frac{\partial \psi_{k}^{(1)}}{\partial u}+\alpha_{k} \frac{\gamma}{u^{3}} \psi_{k}^{(1)}=\frac{2 k+1}{2} \int_{-1}^{1} P_{k}(\mu) \frac{\partial}{\partial u_{\|}} \psi^{(0)} \equiv b_{k}(\tau . u),
$$

where we defined, for notational convenience, the inhomogeneous terms, $b_{k}$. This equation is to be solved for homogeneous initial conditions. A quick solution to the $v_{k}^{(1)}$ is obtainable, because, as we later show, the $b_{k}$ can be put into a much simplified form. The characteristics here are the same as solved for in Eqs. (2.5a) and (2.5b). The equation for $L_{k}^{(1)}$, which is analogous to the homogeneous $\mathrm{Eq} .(2.5 \mathrm{c})$ for $\psi_{k}^{(0)}$, is:

$$
\frac{\partial v_{k}^{(1)}}{\partial s}+\frac{\gamma}{u^{3}} \alpha_{k} w_{k}^{(1)}=b_{k}(s, r)
$$

The solution to this equation, for homogeneous initial conditions. is

$$
w_{k}^{(1)}(s, x)=\left[\frac{1+y(u)}{u}\right]^{\alpha *} \int_{0}^{s}\left[\frac{u}{1+\gamma(u)}\right]^{a k} b_{k}(s, x) d s .
$$


where. $u=u(s . x)=g^{-1}[s+g(x)]$.

It might be imagined that each $b_{k}$ can be expressed only in terms of all the $t_{k}^{(0)}$ : fortunately, bowever. it turns out. as shown in App. $A$, that the $b_{k}$ can be written as the finite composition of the two harmonics. $k-1$ and $k+1$. namely

$$
b_{k}=\frac{k+1}{2 k+3}\left(\frac{\partial}{\partial u} \dot{\varphi}_{k+1}^{(0)}+\frac{k+2}{u} \psi_{k+1}^{(0)}\right)+\frac{k}{2 k-1}\left(\frac{\partial}{\partial u} \psi_{k-1}^{(0)}-\frac{k-1}{u} \dot{t}_{k-1}^{(0)}\right) .
$$

where the partial derivative with respect to $u$ is at constant $\tau$. For insertion into Eq. (2.10), we need the $b_{k}$, and hence $\partial u_{k}^{(0)} / \partial u$, as functions of $x$ and $s$. First note that

$$
\frac{\partial}{\partial u}=\frac{\partial x}{\partial u} \frac{\partial}{\partial x}+\frac{\partial s}{\partial u} \frac{\partial}{\partial s}
$$

Since, from Eq. (2.5), $s(u, \tau)=\tau$, we have $\partial s / \partial u=0$. From Eq. $(2.7)$, we have

$$
\left.\frac{\partial x}{\partial u}\right|_{r}=\frac{g^{\prime}(u)}{g^{\prime}(x)}
$$

where $g^{\prime}(u) \equiv d g / d u=u^{2} / \gamma^{2}(u)$. Thus, at constant $\tau, \partial / \partial u=\left[g^{\prime}(u) / g^{\prime}(x) \mid \partial / \partial x\right.$, and conversely, at constant $s, \partial / \partial x=\left[g^{\prime}(x) / g^{\prime}(u)\right] \partial / \partial u$. Note also that, from Eq. (2.5c), $w_{k}^{(0)}$ separates into

$$
\psi_{k}^{(0)}=I_{k}(x) Y_{k}(x) / Y_{k}(u)
$$

where $x=g^{-1}[g(u)-\tau]$ and

$$
Y_{k}(x) \equiv\left(\frac{x}{1+\gamma(x)}\right)^{0 .}
$$

Note also that $d Y_{k}(x) / d x=\alpha_{k} Y_{k}(x) / x \gamma(x)$. Using Eqs. (2.12)-(2.15), we can write

$$
\frac{\partial \psi_{k}^{(0)}}{\partial u}=\frac{g^{\prime}(u)}{g^{\prime}(x)} \frac{Y_{k}(x)}{Y_{k}(u)}\left\{\frac{d}{d x} Y_{k}(x)+\alpha_{k} I_{k}(x)\left[\frac{1}{x \gamma(x)}-\frac{1}{u \gamma(u)} \frac{g^{\prime}(x)}{g^{\prime}(u)}\right]\right\} .
$$

Inserting Eq. (2.16) into Eq. (2.11), we have the $b_{k}$ in terms of $s$ and $u$ only. We now obtain $\psi_{k}^{(1)}$ by integrating Eq. (2.10).

The integral over $s$ is to be performed at constant $x$. Since $g(u)=g(x)+s$. we can change variables, $d s=g^{\prime}(u) d u$. Lising $x$ constant in Eqs. $(2.10)$, we have $u=x$ at $s=0$. so that, in changing variables, the range is now from $x$ to $u$. and the integral in Eq. (2.10). again perforned at constant $x$, is rewritten as

$$
u_{k}^{(1)}(s . x)=\frac{1}{Y_{k}(u)} \int_{0}^{s} Y_{k}(u) b_{k}(s, x) d s=\frac{I}{Y_{k}(u)} \int_{x}^{*} Y_{k}(u) b_{k}(g(u)-g(x), r) g^{\prime}(u) d u \text {. }
$$

From Eqs. (2.5a) and (2.7), we have $\psi_{k}^{(1)}(u, \tau)=u_{k}^{\prime(1)}(s(u, r), x(u, \tau)]$. Given the response function $\psi_{k}$, we can of course write the radiation response $R(\omega, r)$. Expanding $Q(u)$ and 
$\psi$ in Eq. (1.3) into Legendre components $Q_{k}(u)$ and $\psi_{k}$, and performing the $\mu$-integration. we get

$$
R(\omega, \tau ; \theta)=\sum_{k} \frac{4 \pi}{2 k+1} \int_{0}^{\infty} u^{2} d u \psi_{k}(\omega, u, \tau ; \theta) Q_{k}(u)
$$

The solution, $\psi_{k}=\psi_{k}^{(0)}+\psi_{k}^{(1)}$, is written in complete form in Appendix B, where some further simplifications are noted in obtaining $R(\omega, \tau)$ from the $\psi_{k}$. Incidentally, note that at $\tau=0, I=u$, so $\psi_{k}^{(1)}(u, \tau=0)=0$, since the range of integration in Eq. (2.17) shrinks to zero. The response first order in the electric field vanishes at $\tau=0$ because. as we might expect, the electric field does not immediately cause a large deviation in the electron trajectory; rather, it is the cumulative effect over time of the acceleration due to the field that leads eventually to large differences in the radiation response.

\section{Loop-Voltage Tomography}

In order to examine the sensitivity of the radiation response to important plasma parameters, we simulate an example of practical value. The example illustrates three points: first, that the synchrotron radiation dat a can be quite informative - in this example. an interior dc electric field can be deduced in a tokamak; second, that a parameter space of several dimensions can be scanned numerically quite efficiently - here, response patterns produced by over a million parameter sets spanning a six-dimensional space are compared to the data; third, that the six-dimensional parameter space nearly separates into smaller partitions, in the sense that the data depend on the parameters in ways different enough that the parameters can be inferred independently.

The example that we set is that one detector is available, which observes along a vertical direction (at constant $B_{\text {tor }}$ ), so that the detector sums radiation arising from points all along the viewing direction. The incremental radiation might arise from a brief surge of lower-hybrid power - the background radiation is ignored. We use here a very coarse-grained model in which radiation originates at just two points along the viewing directiois, one point in the plasma center, and one peripheral point (see Fig. 2). While we imagine that the plasma is well-characterized in other ways, we do imagine that we are unsure as to how large a perturbation, $A$, was created at each of the two points. We also imagine that we are unsure of the $q$ profile, which means that we must also treat as unknown the viewing angle with respect to the magnetic field at each of these points. Given these unknowns, we wish to determine the dc parallel electric field at each point. We do imagine that the ion charge state is known and the same at each of the two points, and that the density at each point is known and different. Suppose further that the location in velocity space of the absorbed probing radiation is also known and the same at each point, possibly because of a resonance condition. Thus the detector surns

$$
R(\omega, t)=R\left(\omega, t \mid n_{c}, Z_{\mathrm{eff}}: \varepsilon_{c}, A_{c}, \theta_{c}\right)+P\left(\omega, t \mid n_{p}, Z_{\mathrm{eff}} ; \mathcal{E}_{\mathrm{p}}, A_{p}, \theta_{p}\right),
$$

where $c$ labels parameters at the plasma center and $p$ labels parameters at a peripheral point. Thus. the problem is to find the probability distribution over all possible parameter jets in the six-dimensional space $\left(\mathcal{E}_{c}, \mathcal{A}_{c}, \theta_{c}, \mathcal{E}_{p}, \mathcal{A}_{p}, \theta_{p}\right)$ given a very crude a priori probability distribution and the data $R(\omega, t)$. In Fig. 1, we show the incremental radiation 
response for two different electric fields. What is observed here, however, is only the sum of two such responses, and the object is to deduce the separate contributions to this sum in the presence of noise.

Sote that if the density were the same at the two radiating points, then there would be no way to distinguish radiation emanating from the plasma center from radiation emanating from the peripheral point, no matter how different the other parameters might be. The densily difference means that radiation from the denser place (generally but not necessarily the center) decays faster. Although only the sum of the radiation from the two points is measured, the different decay constants distinguish the contributions to this sum. The larger the density difference, the easier the distinguishing of the individual contributions.

We simulate the results of an experiment by calculating first the radiation response $R(\omega, t)$ in Eq. (3.1) for a specific set of plasma parameters. We assume, however, that in a realistic experiment this precise radiation response is not measured directly. e.g., because of calibration errors, because of background radiation fluctations, or because of imprecise assumptions concerning the governing physics. For the purposes of this simulation, we model all of these uncertainties by gaussian uncorrelated noise, i.e, we imagine that we measure instead $R_{z}(\omega, t)=R(\omega, t)+\sigma(\omega, t)$, where $\sigma$ is an uncorrelated noise signal. In our work, we shali for simplicity assume time and frequency independent noise, $\sigma(\omega, t)$ to be a constant. Obviously, in the limit $\sigma \rightarrow \infty$, there is total degradation of our measurement, and we are left with the a priori probabilities as our best guess for the parameter set probabilities. For finite $\sigma$, our guess can be far more informed. Given the noise model, we can, of course, make precise statements concerning the probability distribution of parameters given the noisy data.

As shown by FISCH AND KRITZ (1989a), the probability of deducing parameter set $\{\Theta\}$. given that the data were obtained with parameter set $\left\{\Theta_{d}\right\}$ and under noisy conditions characterized by $\sigma$, can be constructed as an ensemble average

$$
P\left(\Theta \mid \Theta_{d} ; \sigma\right)=\lim _{N_{R} \rightarrow \infty} \frac{1}{N_{R}} \sum_{j=1}^{N_{R}} P\left(\Theta \mid R_{x}^{(j)} ; \sigma\right)
$$

The ensemble average $\left(N_{R} \sim 80\right.$ generally suffices $)$ is over the probability of parameter set $\{\Theta\}$ given both the a priori probability $P(\Theta)$ of the parameter set and the $j$ th pattern of observations $R_{x}^{(j)}$ under conditions $\left\{\Theta_{d}\right\}$. We can put the right-hand side of Eq. (3.2) in terms of known or calculable quantities by using Baves's theorem in the form $P\left(\Theta \mid R_{x}\right)=$ $P\left(R_{x} \mid \Theta\right) P(\Theta) / P\left(R_{x}\right)$. We have $P\left(R_{x}\right)=\sum P\left(K_{x} \mid \Theta\right) P(\Theta)$, where the sum is $c$ 'er all possible parameter sets $\{\Theta\}$. Also, the probability density of observing the data set pattern $R_{x}$. given the paraneter set $\{\Theta\}, P\left(R_{x} \mid \Theta\right)$, can be written as

$$
P\left(R_{x} \mid \Theta\right) \equiv P\left[\left\{R_{x}\left(\omega_{i}, \tau\right)\right\} \mid \Theta\right]=\prod_{i=1}^{N_{D}} \frac{1}{\sqrt{\pi} \sigma} \exp \left[-\frac{\left[R_{x}(\omega, \tau)-R(\omega, \tau ; \Theta)\right]^{2}}{\sigma^{2}}\right]
$$

where the data are taken at some set of frequencies and times $\left\{w_{1}, \tau_{3}\right\}$, numbering say $Y_{D}$ data measurements in all. The probability of the complete pattern is the product 
of the probabilities of each datum, since we have assumed that the noise is gaussian and uncorrelated.

If it were certain that measurements were obtained in the complete absence of noise. i.e., $\sigma \rightarrow 0$, then even one measurement $R_{x}\left(\omega_{i}, \tau_{i}\right)$ differing from the expected measurement $\left[R_{r}\left(\omega_{i}, \tau_{i}\right) \mid \Theta\right]$ for the parameter set $\{\Theta\}$ immediately rules out that parameter set $\{\Theta\}$ as possibly explaining the data. In the opposite limit of utter noise, $\sigma \rightarrow \infty$, all measurements are equally likely for any data set, so no particular set of parameters is the preferred explanation of the data. Of course, for finite noise, there is no guarantee that any parameter set $\{\Theta\}$ can reproduce exactly a particular nojsy observation $R_{r}$.

We show in Fig. 3a the marginal probability of deducing the electric fields given the data,

$$
P_{M}\left(\mathcal{E}_{c}, \mathcal{E}_{p}\right) \equiv \sum_{A_{c}, A_{p}, \theta_{c}, \theta_{p}} P\left(\mathcal{E}_{c}, \mathcal{E}_{p}, A_{c}, A_{p}, \theta_{c}, \theta_{p}\right)
$$

where the right-hand side above is calculated using Eq. (3.2). Here, the a priori probability is taken as flat over the range plotted, so that, clearly, the noisy data allow a significant refinement of the a priori probabilities. In this example, we measure radiation at the extraordinary polarization, and the true plasma parameters include a nondimensional dc electric field of 0.08 on axis, 0.0 off axis, and equal perturbation strengths $A$ at both locations, corresponding to a loop voltage on axis not yet relaxed via magnetic diffusion. The true viewing angles are both taken to be perpendicular, $\theta_{p}=\theta_{c}=0$. The density off axis is taken as $60 \%$ of the density on axis. The noise level, $\sigma$, is $10 \%$ of the maximum of the incremental pattern $R(\omega, t)$. The data were simulated on a $40 \times 40$ grid in frequencytime space. The marginal probability distribution was calculated by comparing to the noisy data racliation responses arising from over $1.4 \times 10^{6}$ sets of plasma parameters. In practice, purely experimental noise can be kept much lower.

Of particular interest is to compare the above result to the marginal probability distribution for the electric fields given the correct viewing angles, $P_{M}\left(\mathcal{E}_{c}, \mathcal{E}_{p} \mid \theta_{c}, \theta_{p}\right)$, which is shown in Fig. 3b. (This distribution is the sum, over all possible combinations of $A_{c}$ and $A_{p}$, of the joint probability of both fields and both amplitudes, given the true angles $\theta_{c}=\theta_{p}=0$.) Apparently, the probability distribution for $\left(\varepsilon_{c}, \mathcal{E}_{p}\right)$ is not materially affected by our knowledge concerning the viewing angle; in either event, it is possible to discern the case at hand, where a loop voltage is induced in the plasma center but not at the plasma periphery.

Knowledge of the viewing angle is not critical in determining the electric fields not because the viewing angle is not an important factor in the radiation response, but rather because small changes in the viewing angles affect the radiation response in ways very different than do small changes in the electric fields. This can be seen by considering the marginal probabilities of the viewing angles, $P_{M}\left(\theta_{c}, \theta_{p}\right)$, which we depict in Fig. 4 . The viewing angles, from which we could deduce the $q$-profile, are sach resolved on the order of $\pm 1^{\circ}$. Clearly, in order to deduce so well the viewing angles from the radiation, the radiation must, in fact, be sensitive to these angles as well as to the electric field, but the deduction of these parameters from the radiation can proceed almost orthogonally. Alternatively, one can sum over all possible viewing angles and all possible electric fields at the periphery, as well as over both radiation amplitudes, to find the marginal probability 
$P_{M}\left(\mathcal{E}_{c}, \theta_{c}\right)$, which we show in Fig. 5. Again, the marginil probability distribution is fairly sharp. The sharpness of the probability distribution is not very much degraded from what one might obtain were the radiation in fact emanating from only one point along the viewing direction, again indicating a certain orthogonal dependence of the radiation pattern on different plasma parameters. These findings are consistent with what we have found in previous work ( $F$ tSCH AND KRITZ, 1989a), that did not include the loop roltage. but did check for the relative orthogonality of parameters such as $Z_{\text {eff }}, \theta$, and parameters characterizing the probe spectrum.

\section{Information-theoretic Entropy}

These ideas concerning the informative content of the data and the orthogonality of the parameters can be made more precise. Consider the information-theoretic uncertaincy. or entropy, defined by $H \equiv-\sum_{i} P_{i} \log P_{i}$ (see, e.g., Shanson and WeAver, 1949). The entropy is maximum when the probability $P_{i}$ is flat over the allowable space, which is the distribution we take for the a priori probability distribution. The allowable parameter space is assumed to be discrete and finite, but a generalization of these ideas to continuous parameters is possible. A measure of the utility of the data is the reduction in this uncertainty given the data. To this end, define $H_{A}$ as the maximal, a priori, uncertainty; for example, for $N$ possible sets of parameters, we would have $H_{A}=\log N$. A measure of the juformation content in our refined estimate of the probability distribution, say, $P_{M}\left(\mathcal{E}_{c}, \mathcal{E}_{p}\right)$ in $\mathrm{Eq} .(3.4)$ is the uncertainty given the data,

$$
H\left(\mathcal{E}_{c}, \mathcal{E}_{p}\right) \equiv-\sum_{\mathcal{E}_{c}, \mathcal{E}_{p}} P_{M}\left(\mathcal{E}_{c}, \mathcal{E}_{p}\right) \log P_{M}\left(\mathcal{E}_{c}, \mathcal{E}_{p}\right)
$$

The relative uncertainty, $S\left(\mathcal{E}_{c}, \mathcal{E}_{p}\right) \equiv H^{\prime}\left(\mathcal{E}_{c}, \mathcal{E}_{p}\right) / H_{A}$, is a measure of the utility of the data. Here, $H_{A}=\log N$, where $N$ is the number of allowed combinations of $\left(\varepsilon_{c}, \varepsilon_{p}\right)$. A relative uncertainty $S=1.0$ corresponds to the data being entirely useless, whereas the limit $S=0.0$ corresponds to the data being entirely conclusive. The relative uncertainty $S\left(\mathcal{E}_{\mathrm{c}}, \mathcal{E}_{p}\right)$ for the probability distribution in $\mathrm{Fig} .3 \mathrm{a}$ is about 0.66 , which is a considerable reduction from the a priori relative uncertainty of 1 . Similar observations can be made concerning the other parameters. The relative uncertainty for the probability distribution shown in Fig. 4 is $S\left(\theta_{c}, \theta_{p}\right)=0.53$, and for the distribution shown in Fig. 5 , the relative uncertainty is $S\left(\mathcal{E}_{\mathrm{c}}, \theta_{\mathrm{c}}\right)=0.61$.

While these numbers for the relative uncertainty indicate a significant reduction in tncertainty in view of the data, it should be recognized that the meaning of this reduction in uncertainty involves an understanding of how this information might be used. since the exact value of $S$ is dependent on the particular choice of the range of the plasma parameters. A larger range for these parameters, for example, appears to diminish further the relative uncertainty, since coarse discriminations are easier to accomplish than are fine discriminations. Therefore, meaningful comparisons can be made only for the same parameter range. In Fig. 6 we show the relative uncertainty $S\left(\mathcal{E}_{\mathrm{c}}, \mathcal{E}_{\mathrm{p}}\right)$ vs. noise $\sigma$ for peripheral densities at different fractions of the central density. This shows that larger density differences allow finer distinctions to be made in the data. Note two important 
limits. At large noise levels, all data become useless as $S \rightarrow 1.0$. In the limit of varishing noise, all data are conclusive as $S \rightarrow 0.0$.

A measure of what we have called the orthogonality of the plasma parameters is the ,nutual information between them; for example, the mutual information between the fields $\left\{\varepsilon_{c} . \varepsilon_{p}\right\}$ and the viewing angles $\left(\theta_{c}, \theta_{p}\right\}$ is

$$
M\left(\mathcal{E}_{c}, \mathcal{E}_{p i} \theta_{c}, \theta_{p}\right) \equiv H\left(\mathcal{E}_{c}, \mathcal{E}_{p}\right)+H\left(\theta_{c}, \theta_{p}\right)-H\left(\mathcal{E}_{c}, \mathcal{E}_{p}, \theta_{c}, \theta_{p}\right) \geq 0
$$

where the symmetry, $M\left(\mathcal{E}_{c}, \mathcal{E}_{p} ; \theta_{c}, \theta_{p}\right)=M\left(\theta_{c}, \theta_{p} ; \mathcal{E}_{c}, \mathcal{E}_{p}\right)$ follows from the symmetric form of $\mathrm{Eq} .(4.2)$, and the nonzero nature of the mutual information follows from the definitions of the entropies given by Eq. (4.1). The mutual information can be interpreted most easily by defining an average conditional entropy

$$
I\left(\mathcal{E}_{c}, \mathcal{E}_{p} ; \theta_{c}, \theta_{p}\right) \equiv-\sum_{\theta_{c}, \theta_{p}} P_{M}\left(\theta_{c}, \theta_{p}\right) \sum_{\mathcal{E}_{c}, \mathcal{E}_{p}} P_{M}\left(\mathcal{E}_{c}, \mathcal{E}_{p} \mid \theta_{c}, \theta_{p}\right) \log P_{M}\left(\mathcal{E}_{c}, \mathcal{E}_{p} \mid \theta_{c}, \theta_{p}\right)
$$

so that we can rewrite

$$
M\left(\mathcal{E}_{c}, \mathcal{E}_{p} ; \theta_{c}, \theta_{p}\right)=H\left(\mathcal{E}_{c}, \mathcal{E}_{p}\right)-I\left(\mathcal{E}_{c}, \mathcal{E}_{p} ; \theta_{c}, \theta_{p}\right)
$$

Here, we subscript the marginal probability $P_{M}$ to indicate that the amplitudes (and other dependencies not appearing explicitly) have been summed over. Thus, the mutaal information can be interpreted as the uncertainty in $\left(\mathcal{E}_{c}, \mathcal{E}_{p}\right)$ resolved by knowing $\left(\theta_{c}, f_{p}\right)$. There are two important limits here: For independent parameters, i.e., $P_{M}\left(\mathcal{E}_{c}, \mathcal{E}_{p}, \theta_{c}, \theta_{p}\right)=$ $P_{M}\left(\varepsilon_{c}, \mathcal{E}_{p}\right) P\left(\theta_{e}, \theta_{p}\right)$, we have $M\left(\varepsilon_{e}, \varepsilon_{p} ; \theta_{e}, \theta_{p}\right)=0$, i.e., there is no toutual information, so knowing $\left(\theta_{c}, \theta_{p}\right)$ is not helpful in deducing $\left(\mathcal{E}_{c}, \mathcal{E}_{p}\right)$ and rice versa. In the limit that $\left(\theta_{c}, \theta_{p}\right)$ completely determines $\left(\mathcal{E}_{c}, \mathcal{E}_{p}\right)$, then $M\left(\mathcal{E}_{c}, \mathcal{E}_{p} ; \theta_{c}, \theta_{p}\right)=H\left(\varepsilon_{c}, \mathcal{E}_{p}\right)$, so that there is sufficient mutual information to resolve completely the uncertainty in $\left(\mathcal{E}_{\mathrm{c}}, \mathcal{E}_{\mathrm{p}}\right)$. Conversely, in the limit that $\left(\mathcal{E}_{c}, \mathcal{E}_{p}\right)$ completely determines $\left(\theta_{c}, \theta_{p}\right)$, then $M\left(\theta_{c}, \theta_{p} ; \mathcal{E}_{c}, \mathcal{E}_{p}\right)=H\left(\theta_{c}, \theta_{p}\right)$, so that there is sufficient mutual information to resolve completely the uncertainty in $\left(\theta_{c}, \theta_{p}\right)$. Note that since $M\left(\mathcal{E}_{c}, \mathcal{E}_{p} ; \theta_{c}, \theta_{p}\right) \leq H\left(\mathcal{E}_{c}, \mathcal{E}_{p}\right), H\left(\theta_{c}, \theta_{p}\right) \leq H_{A}$, we note that the ratio $M / H_{A}$ varies between 0 and 1 .

Using $\mathrm{Eq},(4.2)$, we have for the mutual information $M\left(\mathcal{E}_{c}, \mathcal{E}_{p} ; \theta_{c}, \theta_{p}\right)=0.05 H_{A}$, for the example given in Sec. 3. This indicates that the uncertainty in $\left(\mathcal{E}_{e}, \mathcal{E}_{\mathrm{p}}\right)$, resolved by knowing $\left(\theta_{c}, \theta_{p}\right)$, is rather small. These numbers make more precise our observation that the data are quite informative in deducing the electric fields in the absence of definite information concerning other plasma parameters, and the further knowledge of these parameters is only of marginal value. We are led to conclude that these parametric dependencies on the radiation reponse are relatively orthogonal. From the standpoint of information processing, we prefer, of course, that the mutual information be small, because this simplifies our scanning of pararneter space. If knowledge of one parameter is not helpful in searching for another, then we can partition the parameter space and conduct independent searches for each independent parameter or each set of only mutually dependent parameters.

The worth of precise information concerning certain parameters in deducing others may also be seen in a somewhat different way. The relative uncertainty $S\left(\mathcal{E}_{c}, \mathcal{E}_{p}\right)$ for the 
probability distribution in Fig. $3 \mathbf{a}$ is about 0.66 , whereas for the distribution in Fig. 3b, $S\left(\varepsilon_{c}, \mathcal{E}_{p} \mid \theta_{c}=0, \theta_{p}=0\right)=0.63$. In other words, exact knowledge of the viewing angles does not further reduce by much the uncertainty in the fields. Similarly, the relative uncertainty $S\left(\theta_{c}, \theta_{p}\right)$ for the probability distribution in Fig. 4 is 0.53 . whereas, if the electric fields were known precisely, then we would have $S\left(\theta_{c}, \theta_{p} \mid \mathcal{E}_{c}=0.08, \mathcal{E}_{p}=0\right)=0.50$. For the distribution in Fig. 5, the relative uncertainty is reduced only from $S\left(\varepsilon_{\mathrm{c}}, \theta_{c}\right)=0.61$ to $S\left(\mathcal{E}_{c}, \theta_{c} \mid \mathcal{E}_{p}=0, \theta_{p}=0\right)=0.59$, when the peripheral parameters are known. In all cases here, the comparisons are made without precise knowledge of $A_{c}$ and $A_{p}$.

\section{Summary and Extensions}

We present a technique for uncovering plasma parameters by perturbing the highvelocity, superthermal, very fast electrons. These electrons synchrotron radiate copiously. but lose energy slowly, so that there can be many time points in a recognizable incremental radiation pattern $R(\omega, t)$. It is the time information in this pattern that makes it inform on certain plasma parmeters such as the loop voltage or the ion charge state. Eliciting this information relies on, among other things, a clear understanding of the dynamics of these fast electrons.

In this regard, the physics of these electrons is relatively well-founded. Dominated by Coulomb collisions and the de electric fieid, these electrons mainly flow along the magnetic field, largely immune to temperature fluctuations and other turbulence in the bulk of the ion cr electron distributions. This understanding of the dynamics of fast electrons has received considerable experimental verification, particularly in experiments designed to drive current by lower-hybrid waves (JOBES et al, 1985; FISCH AND KARNEY, 1985; KARNEY et al., 1985; TAKASE et al., 1987; LeUTERER et al., 1985). Since relatively few parameters govern the dynamics of these electrons, it is possible to formulate a tractable inverse problem of determining parameters from the radiation response.

Although the model that we employ has been highly successful in describing the current-drive experiments, the conclusion that can be drawn from these experiments is only that the model is successful in an average sense, since current is a cross-section integrated quantity. This means that there is some room to question the experimental basis for the physics model. The primary effect not accounted for in our model is the possibility of cross-field transport of fast electrons due to imperfect magnetic surfaces (STIX, 1978; RECHESTER AND ROSENBLUTH, 1978). On the other hand, at least in the current drive experiments, this effect could not have been too strong, or the current-drive effect would not have been observed. The experiments, however, were carried out over a parameter range that is not necessarily subject to extrapolation. Accounting for the losses of these fast electrons (LUCKHARDT, 1987) might be accomplished analytically by introducing only a few new parameters. Since the radiation respunse remains linear, a Green's function can still be defined and the fast algorithms that we have developed might be generalized to incl-de transport and losses. Of course, it may be that in many instances the model as presented would suffice.

The physics of trapped electrons is also not included in the present model. For probing radiation such as lower hybrid waves that interacts with electrons with high parallel velocity but only average perpendicular velocity, the electrons that carry the incremental signal 
remain largely untrapped as they slow down. The tendency to remain untrapped is more pronounced for the very fast electrons that we consider, because the mildy reiarivistic mass increase favors slowing down in energy over pitch-angle scattering. The trapping effect is ignorable here since. by the time an electron is trapped. it has already slowed down to an energy at which the radiation is small. For probing radiation that interacts with trapped or nearly trapped electrons. however. the analysis here needs to be extended.

The probing radiation must be powerful enough to produce in the plasma distingujshable incremental radiation. The power requirement here is far less than for plasma heating. but may not be entirely negligible. A major source of noise is the fluctuations of the background radiation, so a more powerful incremental signal is desirable. Sote that the total radiation power loss from a single electron scales as $p_{\perp}^{2}$. For a Maxwellian. nonrelativistic distribution, the average radiation is from electrons with $v_{\perp}=\sqrt{2} v_{T}$. Thus. for example. tail electrons comprising only $0.1 \%$ of the electron density. but situated at $v_{\perp}=10 v^{\prime} \mathrm{r}$. would contribute approximately $5 \%$ of the synchrotron radiation. While this is still a smail] part of the background emission, what is important is ihat there are frequency windows in which the incremental radiation dominates. For example, for perpendicular viewing $(\theta=0)$, the background radiation appears primarily at the cyclotron harmonics, whereas the incremental radiation appears between these harmonics. since the fast electrons responsible for the incremental radiation pattern suffer relativistic mass enhancement.

The efficiency of producing the incremental radiation can be defined as the fraction of the incident probing if power that is converted to the incremental signal power. Note that power loss of a single fast electron due to synchrotron radiation or due to collisions can be written as

$$
\begin{aligned}
& P_{\mathrm{syz}}=-\nu_{s y a}\left(p_{\perp}^{2}\right) / m, \\
& P_{\text {col }}=-\nu_{c \gamma c}(m c)^{2} / p,
\end{aligned}
$$

where $v_{c}=\left(\omega_{p} / 4 \pi\right) \ln \Lambda\left(\omega_{y}^{3} / n c^{3}\right), \nu_{y y n}=\left(\omega_{p} / 6 \pi\right)\left(\omega_{c} / \omega_{p}\right)^{2}\left(\omega_{p}^{3} / n c^{3}\right)$, and $\omega_{c}$ is the nonrelativistic cyclotron frequency $q B / m$. Thus, very approximately, the fraction of incident probing radiation that is reflected back as synchrotron radiation is

$$
\frac{P_{\text {yyn }}}{P_{\text {col }}}=\frac{2 \ln \Lambda}{3 \gamma} \frac{\omega_{c}^{2}}{\omega_{p}^{2}}\left(1-\mu^{2}\right)(p / m c)^{3} .
$$

This indicates, e.g., that about $10 \%$ of the absorbed probing radiation is reflected back as incremental syachrotion radiation for electrons at about $600 \mathrm{keV}$, corresponding to electrons in the tail of the distribution function in a $25 \mathrm{keV}$ plasma. A topic of future research might be to improve the accuracy of the Green's function for the radiation response by accounting for the radiation recoil through a further expansion in $P_{\text {syn }} / P_{\text {cal }}$. This refinement would be useful at the high end of energies (radiating electrons at greater than $600 \mathrm{keV}$ ) that this diagnostic might employ.

In our work we considered radiation emanating from only two spatial locations. and we considered only one detector to be available. A question for further pursuit is how best to exploit the happy situation when several views of the tokamak are possible. Also, in a more refined calculation, more spatial locations in the plasma should be considered. In 
practice, however, rather than merely considering a finer mesh of independent radiating points, a functional parameterization of the spatial location of the radiation might be employed. While such an ansatz could significantly reduce the parameter space that need be considered. it remains a topic of further research to determine the extent to which the relatjve orthogonality of the parameters could aid in formulating this ansatz.

In general. there is a great opportunity to limit the computational burden through fea. ture selection. For example. in the tomography problem in Sec. 3, in fact. only amplitudenormalized response patterns were compared. In other words, only shape information was retained, which effectively reduced the parameter space dimerision from six to five (since only the ratio $A_{p} / A_{c}$ enters). No further feature selection was pursued, but. in this example, great use was made of the scale-invariant features of the radiation response given in Eq. (1.4). Although $1.4 \times 10^{6}$ radiation patterns were compared, only 13 were actually computed from the radiation response. These 13-represented the possible viewing angles. The remainder of the responses were obtained from $\mathrm{Eq}$. (1.4), by varying intensity, electric field, or density.

In summary, the relatively modest diagnostic system that we propose includes both the brief, probing if signal that leads to the incremental synchrotion signal, and an array of frequency detectors with submillisecond time resolution. In this manner, a great deal of data is focused on but a few choice parameters, including the otherwise unmeasurable de loop voltage. Powerful analytic tools make feasible a numerical analysis of data that would otherwise be unthinkable. The example that we offer indicates that very large parameter spaces can be scanned efficiently. It is our hope to obtain information that is both novel and useful.

Acknowledgments - This work was supported by the United States Department of Energy under contract numbers DE-AC02-76-CHO3073 and DE-FG02-84-ER53187.

\section{Appendix A. Simpliflcation of Inhomogeneous Terms}

Here, we show that the inhomogeneous terms $b_{k}$ can be written in the simplified form : $\quad$ : given in Eq.(2.11), namely

$$
b_{k}=\frac{k+1}{2 k+3}\left(k+2-\frac{\alpha_{k+1}}{\gamma(u)}\right) \frac{\psi_{k+1}^{(0)}}{u}-\frac{k}{2 k-1}\left(k-1+\frac{\alpha_{k-1}}{u}\right) \frac{\psi_{k-1}^{(0)}}{u} .
$$

The simplification relies, in part, on the derivation, which we proceed to show, of several useful identities involving Legendre polynomials.

Beginning with Eq.(2.8), we have

$$
\begin{aligned}
b_{k}(\tau, u) & \equiv \frac{2 k+1}{2} \int_{-1}^{1} P_{k}(\mu) \frac{\partial}{\partial u_{\|}} \psi^{(0)}=\frac{2 k+1}{2} \int_{-1}^{1} P_{k}(\mu)\left(\mu \frac{\partial}{\partial u}+\frac{1-\mu^{2}}{u} \frac{\partial}{\partial \mu}\right) \sum_{l} P_{l}(\mu) \psi_{l}^{(0)} \\
& =\frac{2 k+1}{2} \sum_{l}\left(A_{k l} \frac{1}{u} \psi_{l}^{(0)}+B_{k l} \frac{\partial}{\partial u} \psi_{l}^{(0)}\right),
\end{aligned}
$$

where we define

$$
A_{k l} \equiv \int_{-1}^{1} P_{k}(\mu)\left(1-\mu^{2}\right) \frac{\partial}{\partial \mu} P_{l}(\mu) d \mu
$$




$$
B_{k l} \equiv \int_{-1}^{1} P_{k}(\mu) \mu P_{I}(\mu) d \mu .
$$

These expressions can be simplified through the use of the Legendre identity

$$
\mu P_{k}=\frac{1}{2 k+1}\left[(k+1) P_{k+1}+k P_{k-1}\right],
$$

so that

$$
\begin{aligned}
B_{k l} & =\frac{1}{2 k+1} \int_{-1}^{1} P_{k}(\mu)\left[(k+1) P_{k+1}+k P_{k-1}\right] P_{l}(\mu) d \mu \\
& =\frac{2}{(2 k+1)(2 l+1)}\left[(k+1) \delta_{l, k+1}+k \delta_{l, k-1}\right] \\
& =\frac{2}{(2 k+1)(2 l+1)}\left[l \delta_{l-1, k}+(l+1) \delta_{l+1, k}\right] .
\end{aligned}
$$

From the last equality, which made use of the definition of the Kronecker delta function $\left(\delta_{i j}=1, i=j ; \delta_{i j}=0, i \neq j\right)$, it is evident that $B_{k i}=B_{l k}$.

To simplify the $A_{k l}$, we can integrate by parts, in the definition (A.3a), to get

$$
\begin{aligned}
A_{k l} & =-\int_{-1}^{1} P_{l}(\mu) \frac{\partial}{\partial \mu}\left[\left(1-\mu^{2}\right) P_{l}(\mu)\right] d \mu \\
& =-\int_{-1}^{1} P_{l}(\mu)\left(1-\mu^{2}\right) \frac{\partial}{\partial \mu} P_{k}(\mu) d \mu+2 \int_{-1}^{1} P_{k}(\mu) \mu P_{l}(\mu) d \mu=-A_{l k}+2 B_{k l} .
\end{aligned}
$$

From the last equality we have

$$
A_{k l}+A_{i k}=2 B_{k l} \text {. }
$$

Next, we note that the $P_{k}(\mu)$ also satisfy the Legendre equation

$$
\frac{\partial}{\partial \mu}\left(1-\mu^{2}\right) \frac{\partial}{\partial \mu} P_{k}(\mu)=-k(k+1) P_{k}(\mu),
$$

so in the definition (A.3a) we could substitute

$$
\begin{aligned}
A_{k l} & =-\frac{1}{k(k+1)} \int_{-1}^{1}\left[\frac{\partial}{\partial \mu}\left(1-\mu^{2}\right) \frac{\partial}{\partial \mu} P_{k}(\mu)\right]\left(1-\mu^{2}\right) \frac{\partial}{\partial \mu} P_{l}(\mu) d \mu \\
& =\frac{1}{k(k+1)} \int_{-1}^{1}\left[\left(1-\mu^{2}\right) \frac{\partial}{\partial \mu} P_{k}(\mu)\right]\left[\frac{\partial}{\partial \mu}\left(1-\mu^{2}\right) \frac{\partial}{\partial \mu} P_{l}(\mu)\right] d \mu \\
& =-\frac{l(l+1)}{k(k+1)} \int_{-1}^{1}\left[\left(1-\mu^{2}\right) \frac{\partial}{\partial \mu} P_{k}(\mu)\right] P_{l}(\mu) d \mu,
\end{aligned}
$$

where we integrated by parts to get the second equality and then used again the Legendre equation (A.S) to get the last equality above. Using now the definition, (A.3a), for $A_{k l}$ in the last term above, Eq. (A.9) gives us

$$
A_{k l}=-\frac{l(l+1)}{k(k+1)} \cdot A_{l k} .
$$


Combining now Eqs. (A.T) and (A.10), we have

$$
A_{k l}\left[I-\frac{l(l+1)}{k(k+1)}\right]=2 B_{k l},
$$

and using Eq. (A.5), we finally get

$$
A_{k l}=\frac{2 l(l+1)}{(2 l+1)(2 k+1)}\left[\delta_{k, l-1}-\delta_{l, k-1}\right] .
$$

Finally, using Eqs. (A.12) and (A.5) in Eq. (A.2), we derive the expression for the $b_{k}$ given in Eq. (A.1).

\section{Appendix B. Radiation Response to First Order}

In this appendix, we present the complete radiation response to first order in the electric feid. Using Eq. (2.16) in Eq. (2.11), we can rewrite

$$
\begin{aligned}
b_{k}= & \frac{k+1}{2 k+3}\left(\frac{k+2}{u}-\frac{\alpha_{k+1}}{u \gamma(u)}++\frac{\alpha_{k+1}}{x \gamma(x)} \frac{g^{\prime}(u)}{g^{\prime}(x)}\right) \psi_{k+1}^{(0)} \\
& +\frac{k}{2 k-1}\left(-\frac{k-1}{u}-\frac{\alpha_{k-1}}{u \gamma(u)}+\frac{\alpha_{k-1}}{x \gamma(x)} \frac{g^{\prime}(u)}{g^{\prime}(x)}\right) \psi_{k-1}^{(0)} \\
& +\frac{k+1}{2 k+3} \frac{g^{\prime}(u)}{g^{\prime}(x)} \frac{Y_{k+1}(x)}{Y_{k+1}(u)} \frac{d}{d x} I_{k+1}(x)+\frac{k}{2 k-1} \frac{g^{\prime}(u)}{g^{\prime}(x)} \frac{Y_{k-1}(x)}{Y_{k-1}(u)} \frac{d}{d x} I_{k-1}(x) .
\end{aligned}
$$

The Green's functions can be found by substituting for $b_{k}$ in Eq. (2.17). The radiation response $R(\omega, \tau)$ is then found by integrating Eq. (2.18). In practice, it is numerically expensive to retain the derivative terms $d I_{k} / d x$, since the $I_{k}$ have many parametric dependencies; however, the contributions of these terms to the radiation response allows a simplification, because of the resonance condition. To keep track of terms, let us separate

$$
\psi_{k}^{(1)}=A I_{k+1}+B \frac{d}{d x} I_{k+1}+C I_{k-1}+D \frac{d}{d x} I_{k-1},
$$

where the coefficients $A, B, C$, and $D$ can be identified through a comparison of Eqs. (B.1) and (B.2). The radiation response can be written as

$$
R(\omega, \tau)=R_{A}+R_{B}+R_{C}+R_{D}
$$

and the subscripts identify the terms in Eq. (B.1) through the corresponding coefficients. The radiation response term $R_{A}$ can be found directly from the corresponding response function

$$
\begin{aligned}
\psi_{k}^{(1 \lambda)}(s, x)= & \frac{(k+1)}{2 k+3} I_{k+1}(x)\left[\frac{Y_{k+1}(x)}{Y_{k}(u)}\right] \\
& \int_{x}^{u}\left[\frac{Y_{k}(u)}{Y_{k+1}(u)}\right]\left(\frac{k+2}{u}-\frac{\alpha_{k+1}}{u \gamma(u)}+\frac{\alpha_{k+1}}{x \gamma(x)} \frac{g^{\prime}(u)}{g^{\prime}(x)}\right) g^{\prime}(u) d u
\end{aligned}
$$


where $x=x(u, \tau)=g^{-1}[g(u)-\tau]$, and one can write

$$
\left[\frac{Y_{k}(u)}{Y_{k+1}(u)}\right]=\left[\frac{1+\gamma(u)}{u}\right]^{\beta_{k}}
$$

with $\beta_{k} \equiv \alpha_{k+1}-\alpha_{k}=\left(1+Z_{\text {eff }}\right)(1+k)$. The Green's function $\psi_{k}^{(1 C)}(s, r)$ can be handied similarly, and the corresponding radiation response is found by direct integration over the Green's function.

In order to simplify, however, e.g., the response function proportional to $d I_{k+1} / d x$, it is expedient to first write the radiation response

$$
R_{B}(\omega, \tau)=\sum_{k} \frac{1}{2 k+1} \int_{0}^{\infty} u^{2} Q_{k}(u) B(u, \tau) \frac{\partial}{\partial u} I_{k+1}(\omega, x) d u
$$

We can rewite

$$
\frac{\partial}{\partial u} I_{k+1}(\omega, x)=\frac{g^{\prime}(x)}{g^{\prime}(u)} \frac{\partial}{\partial u} I_{k+1}(\omega, x)
$$

and then we can integrate Eq.(B.6) by parts, noting that the $Q_{k}(u)$ vanish at the integration limits (because there is no perturbation to the electron distribution function at these energy limits), to obtair

$$
\begin{aligned}
R_{B}(\omega, \tau)= & -\sum_{k} \frac{1}{2 k+1} \int_{0}^{\infty} I_{k+1}(\omega, x) \frac{\partial}{\partial u}\left(u^{2} Q_{k}(u) B(u, \tau) \frac{g^{\prime}(x)}{g^{\prime}(u)}\right) d u \\
= & -\sum_{k} \frac{1}{2 k+1} \int_{0}^{\infty} I_{k+1}(\omega, x)\left\{\left[u^{2} B(u, \tau) \frac{g^{\prime}(x)}{g^{\prime}(u)}\right] \frac{d Q_{k}(u)}{d u}\right. \\
& \left.+Q_{k}(u) \frac{\partial}{\partial u}\left[u^{2} B(u, \tau) \frac{g^{\prime}(x)}{g^{\prime}(u)}\right]\right\} d u .
\end{aligned}
$$

In the second equality above, the bracketed terms are treated as functions of $u$ with $\tau$ entering parametrically. The second of these terms coutributes to $R(\omega, \tau)$ proportional to the initial condition $Q_{k}(u)$ as do the terms $A$ and $D$ in Eq.(B.2). The first bracketed term multiplies $d Q_{k} / d u$, which is numerically easier to store than derivatives of the $I_{k}$, since each of the $Q_{k}$ are just one-dimensional functions, which are given in some analytic form.

The further simplification in determining $R(\omega, \tau)$ accurs upon substitution of the specific form of the radiation function $I_{k}$ which is finite only at the resonance condition. The radiation functions differ somewhat depending upon the polarization observed. For example, for extraordinary polarization (see, e.g., LANDAU AND LIFsHITz, 1951), the radiation intensity $I^{X}$ can be written as

$$
I^{x}(\omega, \theta, \mathbf{u})=\sum_{n} \frac{e^{2} \omega^{2}}{2 \pi c \lambda^{2}}\left(\frac{u}{\gamma}\right)^{2}\left(1-\mu^{2}\right) J_{n}^{\prime 2}\left(\pi \frac{u}{\gamma}\left(1-\mu^{2}\right)^{1 / 2} \frac{\cos \theta}{\lambda}\right) \delta\left(\omega-n \omega_{c} / \gamma \lambda\right)
$$


where $n$ is the cyclotron harmonic, $J_{n}^{\prime}$ is the derivative of the nth Bessel function of the first kind, $\omega_{c}=e B / m c$ is the cyclotron frequency of nonrelativistic electrons, and $\lambda(u, \mu, \theta)=1-u \mu \sin \theta / \gamma$ is the extent of the Doppler shift through viewing the radiation at angle $\theta$. The radiation intensity, for ordinary polarization (i.e., with $\mathbf{E}$ vector parallel to the magnetic field), may be written as

$$
I^{O}(\omega, \theta, \mathbf{u})=\sum_{n} \frac{e^{2} \omega^{2}}{2 \pi c \lambda^{2}}\left(\frac{\sin \theta-u \mu / \gamma}{\cos \theta}\right)^{2} J_{n}^{2}\left(n \frac{u}{\gamma}\left(1-\mu^{2}\right)^{1 / 2} \frac{\cos \theta}{\lambda}\right) \delta\left(\omega-n \omega_{c} / \gamma \lambda\right) .
$$

We now integrate $E q,(B .8)$ and analogous equations for the other response terms over the $\delta$-function resonance in Egs. (B.9). For, say, the ordinary polarization, we get the following equation for the radiation response:

$$
\begin{aligned}
& R(\omega, \tau)=\frac{e^{2} \omega^{2}}{c} \sum_{k} \int_{-1}^{+1} d \mu \sum_{n} \sum_{u_{*}} \frac{1}{2 k+1}\left[\frac{\sin \theta-\rho \mu / \gamma(\rho)}{\cos \theta}\right]^{2}\left|\frac{\partial \omega}{\partial u_{*}}\right|^{-1} \\
& J_{n}^{2}\left(\frac{\omega}{\omega_{c}} \rho \cos \theta \sqrt{1-\mu^{2}}\right)\left[\frac{\rho}{u} \frac{\gamma(u)+1}{\gamma(\rho)+1}\right]^{\alpha_{k}}\left\{Q _ { k } ( u _ { * } ) \left\{P_{k}(\mu)(2 k+1) u_{*}^{2}\right.\right. \\
& +\mathcal{E} P_{k+1}(\mu)(k+1)(k+2) u_{*}^{2}\left[\frac{\rho}{1+\gamma(\rho)}\right]^{\beta_{*}} \int_{\rho}^{u_{*}} d x \frac{x}{\gamma^{2}(x)}\left[\frac{1+\gamma(x)}{I}\right]^{\beta_{k}} \\
& +\mathcal{E} P_{k+1}(\mu)(k+1)\left[\frac{\rho}{1+\gamma(\rho)}\right]^{\theta_{k}}\left\{\left(\alpha_{k} \frac{\gamma(u)}{u}-2 u_{*}\right) \int_{\rho}^{u_{*}} d x \frac{x^{4}}{\gamma^{4}(x)}\left[\frac{1+\gamma(x)}{x}\right]^{\beta_{k}}\right. \\
& \left.-\alpha_{k+1} u_{*}^{2} \int_{p}^{x_{*}} d x \frac{x}{\gamma^{3}(x)}\left[\frac{1+\gamma(x)}{x}\right]^{\beta_{k}}\right\} \\
& -\mathcal{E} P_{k+1}(\mu)(k+1) u_{*}^{2}\left[\frac{u^{2}}{\gamma^{2}(u)}\left[\frac{\rho}{u} \frac{\gamma(u)+1}{\gamma(\rho)+1}\right]^{\beta_{k}}-\frac{\rho^{2}}{\gamma^{2}(\rho)}\right] \\
& -\varepsilon P_{k-1}(\mu)(k+1) k u_{*}^{2}\left[\frac{\rho}{1+\gamma(\rho)}\right]^{-\beta_{t-1}} \int_{\rho}^{\mu *} d x \frac{x}{\gamma^{2}(x)}\left[\frac{1+\gamma(x)}{x}\right]^{-\beta_{k-1}} \\
& +\mathcal{E} P_{k-1}(\mu) k\left[\frac{\rho}{1+\gamma(\rho)}\right]^{-\beta_{k-1}}\left\{\left(\alpha_{k} \frac{\gamma(u)}{u}-2 u_{*}\right) \int_{\rho}^{u} d x \frac{x^{4}}{\gamma^{4}(x)}\left[\frac{1+\gamma(x)}{x}\right]^{-\beta_{k-1}}\right. \\
& \left.-\alpha_{k-1} u_{*}^{2} \int_{p}^{k_{*}} d x \frac{x}{\gamma^{3}(x)}\left[\frac{1+\gamma(x)}{x}\right]^{-\beta_{k-1}}\right\} \\
& \left.-\mathcal{E} P_{k-1}(\mu) k u_{*}^{2}\left[\frac{u^{2}}{\gamma^{2}(u)}\left[\frac{\rho}{u} \frac{\gamma(u)+1}{\gamma(\rho)+1}\right]^{\cdots \beta_{h-1}}-\frac{\rho^{2}}{\gamma^{2}(\rho)}\right]\right\} \\
& -\mathcal{E} \frac{d}{d u}\left[Q_{k}(u)\right]_{u=\pi_{*}} \gamma^{2}(u)
\end{aligned}
$$




$$
\begin{aligned}
& \left\{P_{k+1}(\mu)(k+1)\left[\frac{\rho}{1+\gamma(\rho)}\right]^{\beta_{k}} \int_{\rho}^{u_{k}} d x \frac{x^{4}}{\gamma^{4}(x)}\left[\frac{1+\gamma_{f}(x)}{x}\right]^{\beta_{k}}\right. \\
& \left.\quad+P_{k-1}(\mu) k\left[\frac{\rho}{1+\gamma(\rho)}\right]^{-\beta_{k-1}} \int_{\rho}^{u_{k}} d x \frac{x^{4}}{\gamma^{4}(x)}\left[\frac{1+\gamma(x)}{x}\right]^{-3_{k-1}}\right\}
\end{aligned}
$$

Eq. $\{$ B.10) is in a form suitable for numerical computation; the dummy variable $u$. takes on discrete values, and we used the resonance condition to define a function $u(u) \equiv$ $\omega_{c} / \gamma(u) \lambda(u, \mu, \theta)$. An equation analogous to $\mathrm{Eq} .(\mathrm{B} .10)$ can be written for the extraordinary polarization.

\section{References}

Alikaev V. V., Bobrovskil G. A., Poznyak V. I., Razumova K. A., SANikikov V. V., Sokolov YU. A., and ShMarin A. A. (1976) Fiz. Plazmy 2, 390.

Bornatici M., Cano R., de Barbieri O., and Englemann F. (1983) Nucl. Fusion 23, 1153.

Celata C. M. and BoYd D. (1977) Nucl Fusion 17, 735.

CELATA C. M. (1985) Nucl. Fusion 25, 35.

Fisch N. J. (1987) Rev. Mod. Phys. 59, 175.

Fisch N. J. (1988) Plasma Phys. and Controlled Nucl. Fusion. 30, 1059.

Fisch N. J. and Karney C. F. F. (1985) Phys. Rev. Lett. 54, 897.

FisCh N. J. and KRIT2 A. H. (1989a) to appear in Plaw sa Phys. and Controlled Nucl. Fusion.

Fisch N. J. and Kritz A. H. (1989b) Phys. Rev. Lett. 62, 2393.

GiRuzzi G. (1988) Nucl. Fusion 28, 1413.

Giruzzi G., Fidone I., Granata G., and Krivenski V. (1986) Nucl. Fuvion 26, 662. HUChinson I. H. and Kato K. (1986) Nuei. Fusion 26, 179.

Jobes F. C., Bernabei S., Chu T. K., Hooke W. M., Merservey E. B., Motley R. W., Stevens J. E., and von Goeler S. (1985) Phys. Rev. Lett. 55, 1295.

Karney C. E. F., Fisch N. J., and Jobes F. C. (1985) Phys. Rev. A 32, 2554.

Kato K. and Huchinson I. H. (1986) Phys. Rev. Lett. 56, 340.

LANDAU L. D. and Lifshitz E. M. (1951) Classical Theary of Fields, Addison Wesley Inc., Reading Mass.

LeUTenen F., ECKhartT D., Söldner F., Becker G., BRambilla M., Brinkschulte H., Derfler H., Ditte U., Eberhagen A., Fussmanw G., Gehre O., Gerkhafdt J., Gierke G. v., Glock E., Gruber O., Hass G., Hesse M., JaneSchitz G., Karger F., KeIlhacker M., Kissel S., Klüber O., Kornherr M., Lisitano G., Magne R., Mayer H. M., McCormick K., Meisel D., Mertens V., Müller E. R., Münich M., Murmand H., Poschenrieder W., Rapp H., Ryter F., Schmitter K. H., Schneider F., Siller G., SMeulders P., Steuer IK. H., Vien T., Wagnen F., Woyna F. V., and ZouhaR M. (1985) Phys. Rev. Lett. 55, 75. LUCE T., EFthimion P., and Fisch N. J. (1988) Rev. Sci. Instr. 59, 1593.

LicKHARDT S. C. (1987) Nucl. Fusion 27, 1914.

Mahajan S. M., Oberman C. and Davidson R. C. (1974) Plasma Phys. 16, 1147. 
Shanion C. E. and Wedver W. (1949) The Mathematical Theory of Commurication. University of Illinois Press.

STIX T. H. (1978) Nucl. Fusion 18, 1978.

Takise Y., Kxowlton S., and Ponkolab M. (198T) Phys. Fluids 30, 1169.

TAuOR S. (1979) Nucl. Fusion 19. 455.

\section{Figure Captions}

Figure 1. Radiation response $R(\omega, t)$ (arbitrary units) at extraordinary polarization for different electric fields. (a) normalized electric field $\varepsilon=0.3$ (this corresponds to about $0.02 \mathrm{~V} / \mathrm{m}$ at density $10^{14} / \mathrm{cm}^{3}$ ). (b) $\mathcal{E}=0.0$. The peaks in the plot correspond to second, third, fourth, and fifth harmonic radiation. The incremental radiation arises from electrons initially at about $700 \mathrm{keV}$, corresp unding to tail electrons in a reactor plasma, so the harmonics are relativistically downshifted in frequency. Here, the radiation is viewed perpendicular to the magnetic field and an ion charge state of 1.5 was assumed.

Figure 2. Viewing the radiation. A detector sums radiation emanating from a central region, with electric field $E_{e}$, and from a peripheral region, with electric field $E_{\text {p. }}$. The viewing plane is the vertical plane that is tangent to the magnetic surface, such that, at $\theta=0$, the radiation is viewed perpendicular to the total (toroidal plus poloidal) magnetic field.

Figure 3. Joint rarginal probability distribution. (a) $P_{M}\left(\mathcal{E}_{c}, \mathcal{E}_{p}\right)$. (b) $P_{M}\left(\mathcal{E}_{c}, \mathcal{E}_{p} \mid \theta_{c}, \theta_{p}\right)$. In Fig. $3 \mathrm{~b}$, the marginal distribution was obtained by summing the joint distribution over all combinations of amplitudes $A_{c}$ and $A_{p}$, but using $\theta_{c}=\theta_{p}=0$. For Fig. $3 b$, the relative uncertainty, $S$, is 0.63 , which is only slightly less than for Fig. 3a, where $S$ is 0.66 .

Figure 4. Joint marginal probability jistribution $P_{M}\left(\theta_{c}, \theta_{p}\right)$. The marginal probability distribution was obtained by summing over amplitudes $A_{c}$ and $A_{p}$, and over fields $\mathcal{E}_{c}$ and $\mathcal{E}_{p}$.

Figure 5. Joint marginal probability distribution $\boldsymbol{P}_{M}\left(\mathcal{E}_{c}, \theta_{c}\right)$. The marginal probability distributiuz was obtained by summing over amplitudes $A_{c}$ and $A_{p}$, and over the peripheral parameters $\mathcal{E}_{\mathrm{p}}$ and $\theta_{p}$.

Figure 6. Relative uncertainty given the data, $S\left(\varepsilon_{\varepsilon}, \varepsilon_{p}\right)$, v9. noise level, $\sigma$, for three different ratios of peripheral to central density. 


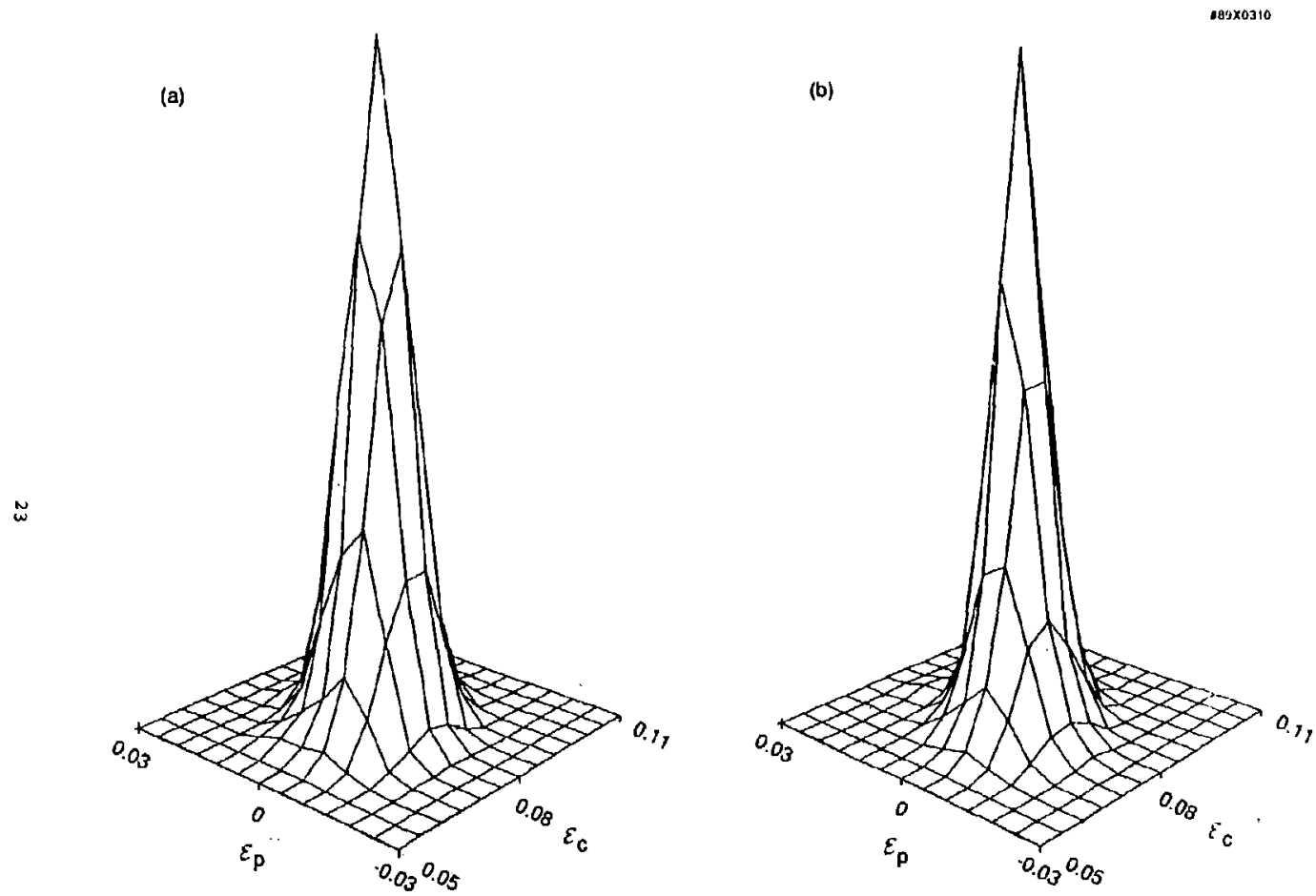

Fiq. 3 


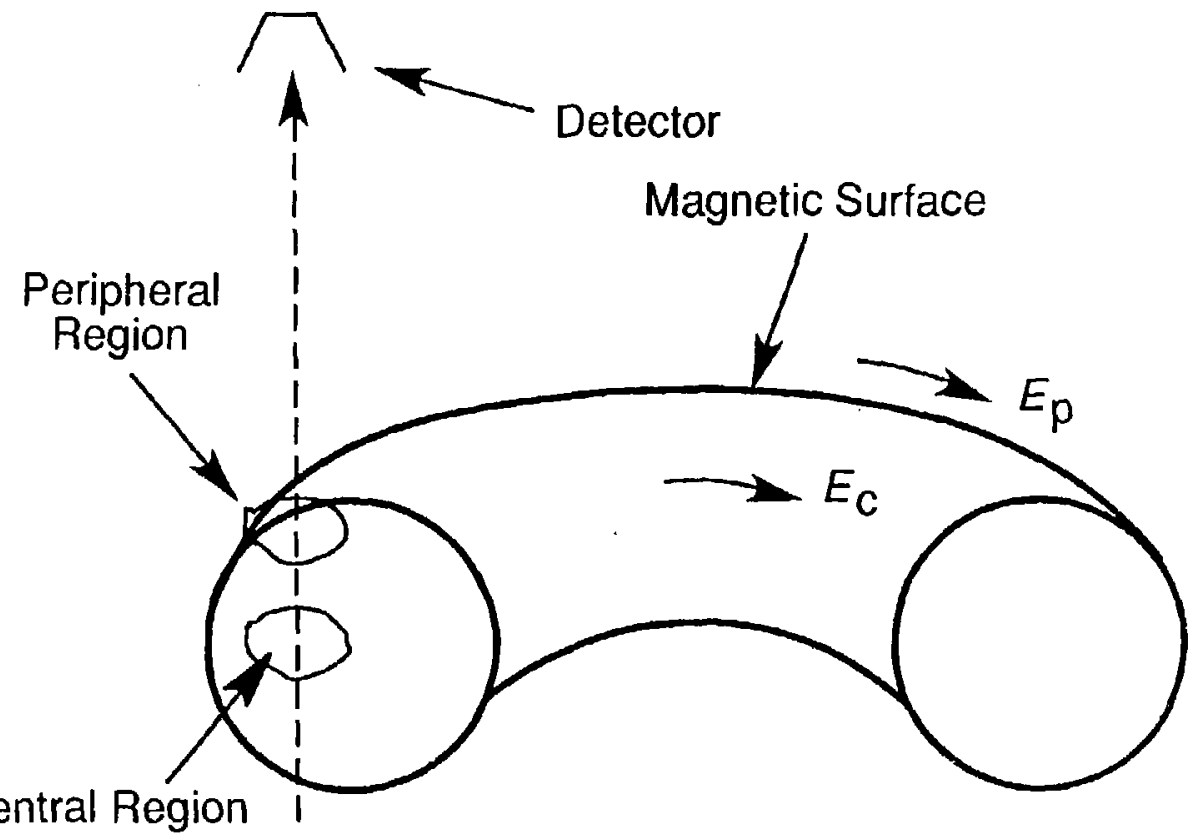

$\therefore$ Central Region

Fig. 2 
$\# 89 \times 0311$

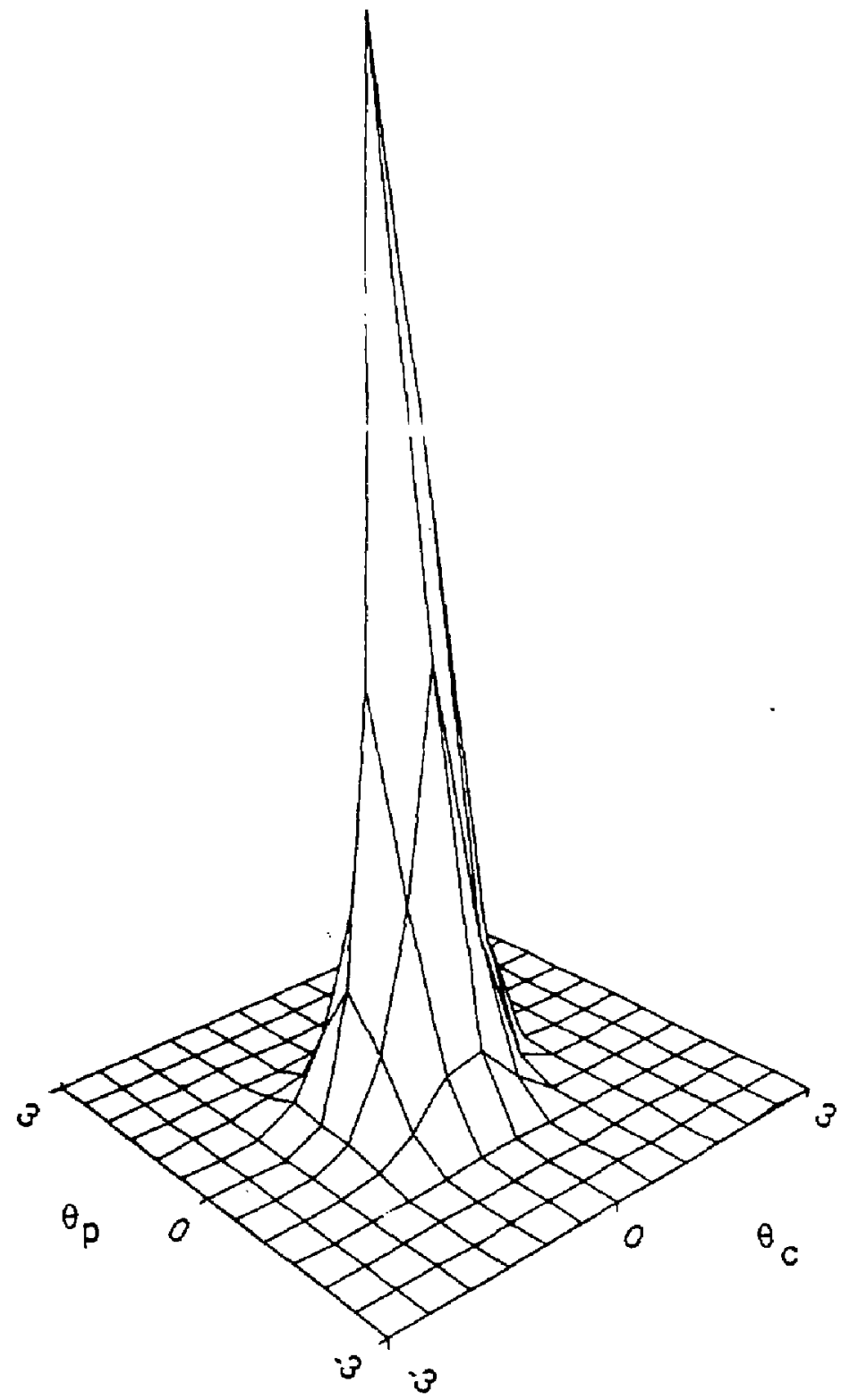

Fig. 4

25 


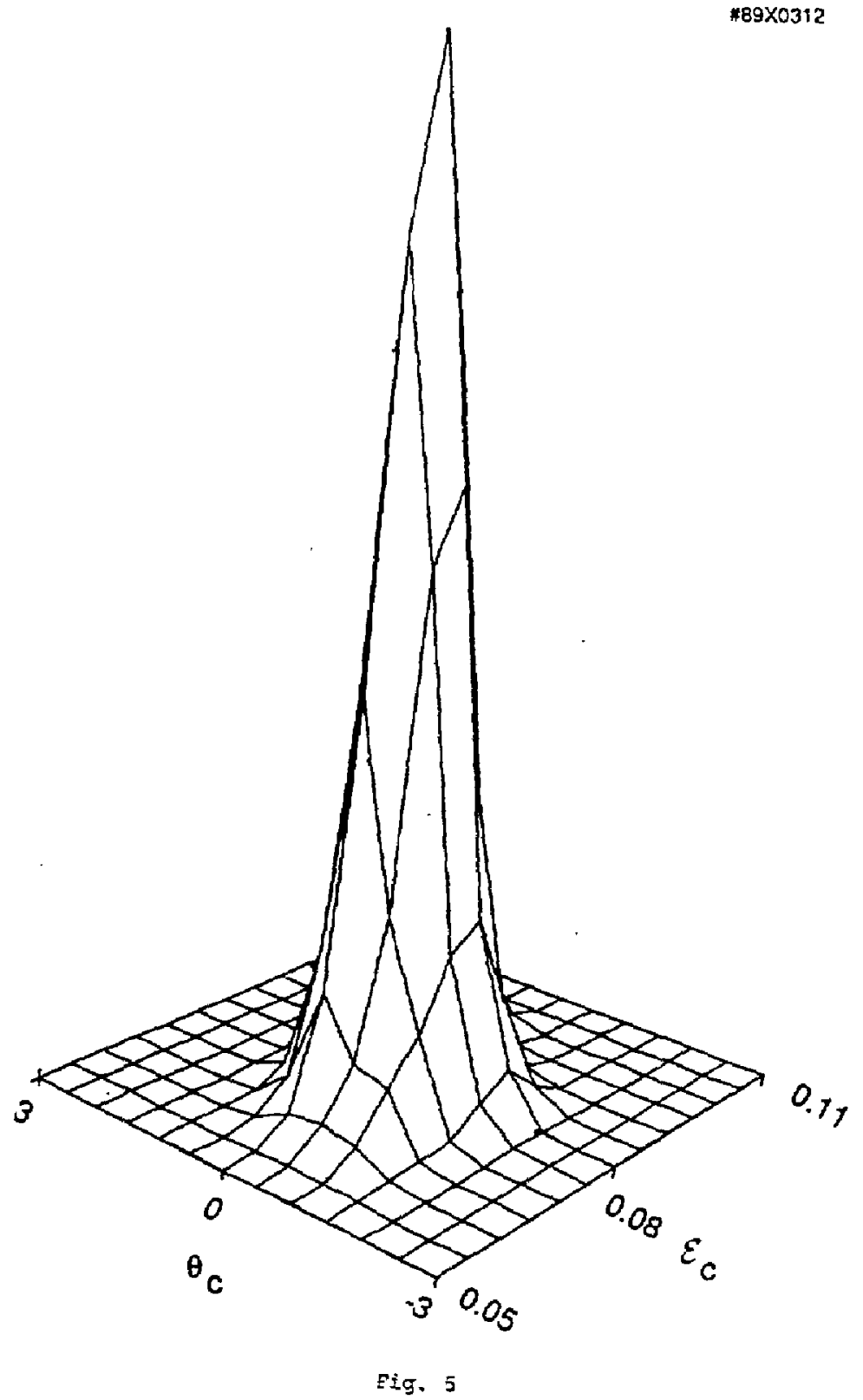




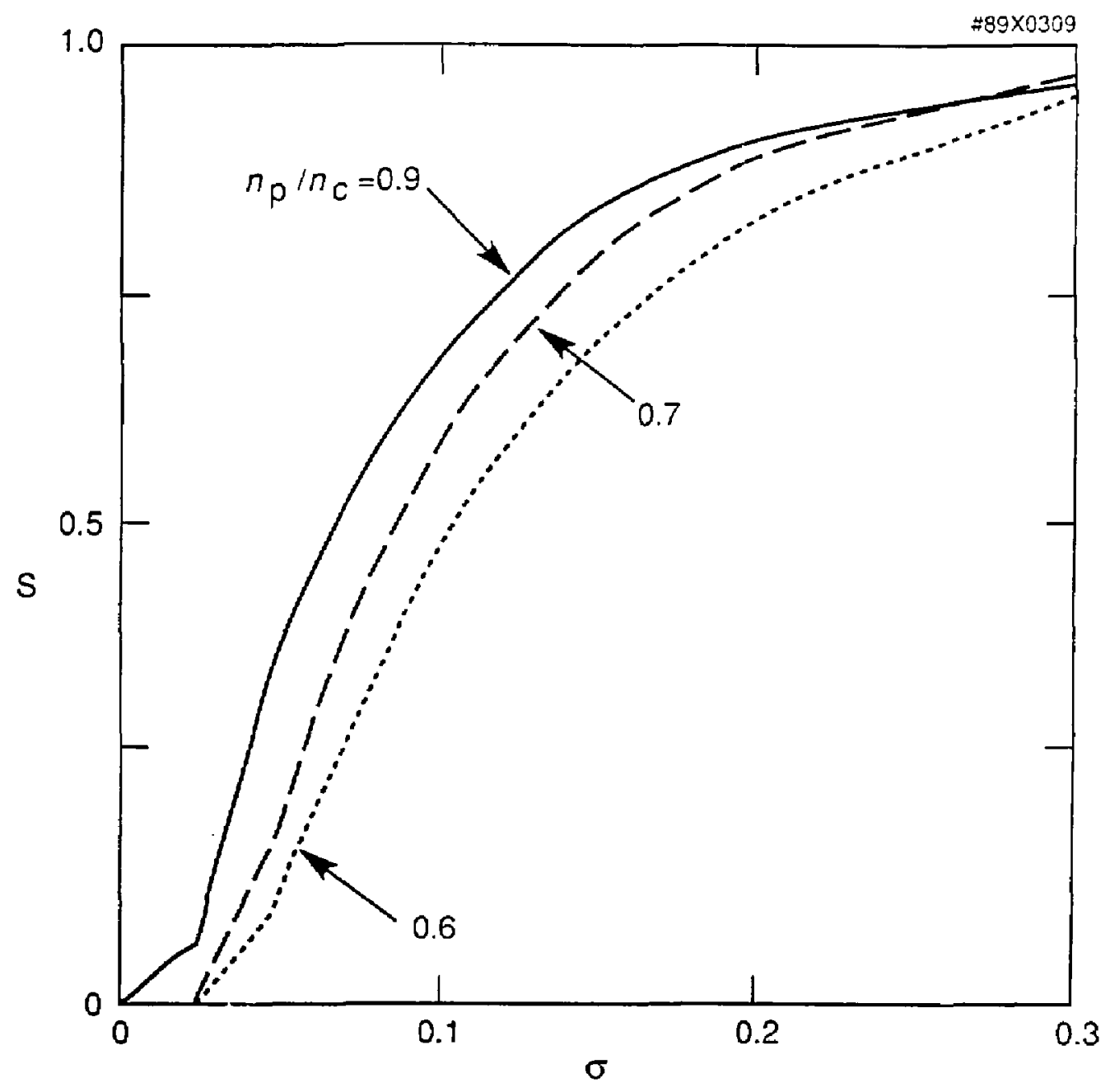

Fig. 6 
Dr. Frank J. Pooloni, Univ of Wollongong. AUSTRALIA Prot. M.H. Brennan, Univ Syoney, AuSTRRLiA Plasme Research Lab., Australiun Not. Univ , AUSTRALIA Prot. I.R. Jones, flinders Univ., Austrull is Prof. F. Cao, Inst Theo Phys, MSTRIa

Prof. M. Hointler, Instut tü Theoratische Physik, AUSTRIA M. Goossans, Astronaliseh Instituut, BeLGium Ecole Royale Militaire, Lo de Phys Plases, Belgium Comission-European, Og-XII Fusion Prog, BeLGILM Prof. R. Boucique, Rljksuniversiteit Gant, Betgrya Dr. P.H. Soksunaka, Instituto Fisice, BazlL Instituto De Pesquisas Espaciesi-IrPE, GAZIL Documents Office, Atanic Energy of Cansda Limited, CAMADn Dr. M.P. Bechynski, Meg Tochnologies, Ine., CAMAd Dr. H.M. Skarsgard, University of Saskatchewen, CwuOA Dr. H. Barnars, University of British Columbia, CavaDA Prot. J. Teichemen, Univ. of Montred, cauba Prot, S.R. Sremivasem, University of Colgary, CAMADA Prof. Tudor M. Johnston, INRS-Energle, Cuwan

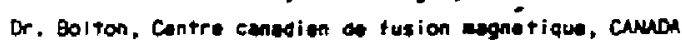
Dr. C.R. Jeens, Uniy. of Alberta, Cuwan

Dr. Pater Luke, Komenskeno Univ, CZECHOSLOYAKIA

The Librarion, Eulnew Laboratory, EMGLAND

The Librarien, Rutherford Appleton Lebor atory, EtoLAND Mrs, S.A. Huteninson. JET Librury, EMGLNAD C. Mouttet, Leb. de Pmysique des Milieux lonisios, FRAnCE J, RAdAT, CEN/CNDARAGE - Bat 506, FRANCE

Ms. C. Rinni, Librarian, Univ. of laannina, GREECE

Dr. Ton Muel, Acedany Bibjiographic Sar., howe kONG

Preprint Librsey, Hungarien Acedemy of Sciences, Mungar

Dr. B. Das Gupta, Sahe Inst of Nue!. Phys.. InDiA

Dr. P. Kow, Instituta for Plased Resemen. IMOIA

Dr. Philio Roseneu, Isreat Inst. of Tech, ISRAEL

Lierarion, Int'I Ctr Theo Phys, ITALY

Prof. G. Aostagni, Istituto Gas Ioniz2ati Del OR, ITALY

Miss Clelid De Polo, Assoc ELRATOM-ENEA, ITALY

Dr. G. Grosso. Istituto di fisico del Plasma, ITALY

Dr. H. Yauato, Toshiba Res \& Dev, JAPAr.
Prot. I. Karakami, Atomic Enargy Res. Institute, JAPAK

Prot. Kyoji Nishikara, Univ of Hiroshima, JAPAN

Director, Dagt. Large Tokomak Res. JAERI, JAPAN

Prot. Satoshi Iton. Kyushu University, JAPAN

Research Info Conter, Nagoyd University. JAPAN

Prof. S. Tanaka, Kyoto University, JaPAN

Librory, Kyoto University, JAPAN

Prot, Nopuyuki Inoue, Univarsity of Tokyo, JAPAN

5. Mori, JAERI, JAPAN

H. Jeong. Librarian, Kored hevanced Enargy Res Inst, KOREA Prot. 0.1. Choi, The Korea Adv. Inst of Sci \& Tech, KOREA Prof. B.5. LIley, University of Waikato, NEW ZEALAND Institute of Plasag Prysics. PEOPLE'S REPUBLIC OF CHINA Librarian, Institute of Phys.. PECPLE'S REPUBLIC OF CHINA Library, Tsing Hua university. PEOPLE'S REPUBLIC OF CHINA 2. Li, Southwest Inst. Physics, PEOPLE's REPUBLIC Of CHIMA Prof. J.A.C. Cabral, Inst Suparior Teenico, PORTUGal Dr. Oetavian Petrus, Al I CuzA Univarsity, ROMANIA Or. Ja de Vililiers, Fusion studies, AEC, SO AFRICA Prot. M.A. Hellberg, University of Natal, SO AFRICA C.I.E.M.A.T., Fusion Div. Liorary, SPAIN Or. Lennart Stenfla, University of UMEA, SWEDEN Liorory, Roysy Institute of Toen, SWEDEN

Prof. Hons Withelmson, Chalears Univ of Tech, SHEDEN Centre Phys das Plasmas, Eeole Polytaeh Fed, 5witzERLANo Biblio"hoek, Fom-lnst Voor Plasma-fysica, THE NETHERLANOS Marin Ourgut, Middle East Teennical University, TUAKEY

Dr. D.D. Ryutow, Siberian Acad Sci. USSR

Dr. G.A. Eliswar, Kurchatov Institute, USSR

Dr. Y.A. Gluknikh, Inst Electrophysical ADDararus, USSR

Prat. 0.5. Padicnenko, Inst, of Phys. o Tech. USSR

Dr. L,M. Kovriznnykn, Institute of Gen. Pnysies, USSR Nuelear Res. Estadishment, Julich Lto., W. GERMANr gioliotnak. Inst. Fur flasnalorschung, w. Gebohany or. K. Scrindier, Ruhr-Universitat bochum, W. GERMANr ASDEX Reading Fon, C10 Wagner, IPPMAox-Planck, W, GEQMANy Librar ian, Has-PIOnek institut. W. GERMANY Prof. R.K. Janey, Inst of Phys, yugoslavia 